\title{
Manual Performance Analysis of a 2.2kw Refurbished Three- Phase Induction Motor
}

\author{
Enyong P.M ${ }^{1}$,Anyaeji C.A ${ }^{2}$ and Izuagie F.I ${ }^{3}$ \\ ${ }^{1,3}$ Department of Electrical and Electronic Engineering, Auchi Polytechnic, Auchi \\ ${ }^{2}$ Department of Electrical and Electronic Engineering, university of benin benin city
}

\begin{abstract}
A refurbished $2.2 \mathrm{~kW}$ 3-phase squirrel-cage induction motor was subjected to a steady-state performance analysis. It was earlier refurbished to reflect a prevailing local maximum 3-phase voltage level of $380 V_{a c}$, as against the manufacturer's name-plate nominal voltage of $415 V_{a c}$. The aim was to obtain from the motor at $380 V_{a c}$ satisfactory values of the operational quantities as obtainable at the original voltage level of $415 V_{a c}$. To this end the method of open-circuit/short-circuit laboratory tests was adopted, the d.c. resistance test notwithstanding. The experimental calculations from these led to the determination of the machine equivalent circuit parameters by means of which the motor operational quantities and the performance curves for analytical purposes were obtained. MATLAB was used to generate the performance curves via the appropriate mathematical models. The analysis showed the motor as exhibiting satisfactory full-load values of torque, speed, output power, slip and power factor, although at the expense of full-load efficiency and starting properties.
\end{abstract}

Keywords: Refurbished Induction Motor, Performance Analysis

\subsection{Introduction}

\section{Introduction \& Motor Data}

Since the advent of rotating machines in 1833 [Say \& Taylor, 1980], the induction motor as a rotating machine has been of immense significance to mankind. When it breaks down under such adverse operational conditions as mechanical overloading, short-circuited turns, single-phasing, under-voltage/over-voltage conditions and ageing, it requires to be refurbished [Mehala N. (2010)]. The machine of interest in this paper is a burnt $2.2 \mathrm{~kW}, 415 \mathrm{~V}$, squirrel-cage induction motor. It got burnt-out due to protracted overheating arising from under-voltage condition of operation overnight. After refurbishment, it became necessary to subject it to a steady-state manual performance analysis (manual in the sense of the absence of computer aid in calculations). Thus, the work in this paper commences proper with the production of the necessary machine equations in Section 2.0. In Section 3.0 the authors shall present tabulated laboratory experimentation results. The experimental calculations from experimentation results and the associated performance curves are dwelt upon in Section 4.0; whilst, in Section 5.0 performance analysis, conclusion and recommendation are dealt with.

\subsection{Motor Data}

The complete machine name-plate data are as tabulated below

Table 1.1: Name-plate Details of the Test Machine

\begin{tabular}{|c|c|c|}
\hline $\begin{array}{l}\text { i) Manufacturer .... BRUSH } \\
\text { ii) Country ..........England } \\
\text { iii) Output power ...2.2kW } \\
\text { iv) Voltage ............415V( }(\Delta)\end{array}$ & 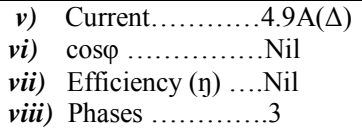 & 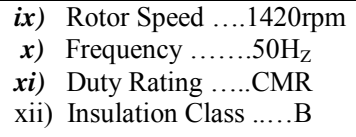 \\
\hline
\end{tabular}

\section{Applicable Machine Equations}

The machine equations applicable for the production of the various quantities and performance curves of the test motor are as summarized below.

2.1 Stator Input Current (On New Voltage Rating), $I_{s(n e w)}$ :

$$
\mathrm{I}_{\mathrm{s}(\text { new })}=\mathrm{I}_{\mathrm{s}(\text { old })} \mathrm{X}\left[\mathrm{V}_{\mathrm{s}(\text { old })} / \mathrm{V}_{\mathrm{s}(\text { new })}\right]
$$

2.2 Effective Load Current, $I_{\mathrm{rs}}$ :

$$
\begin{aligned}
I_{r s} & =\frac{V_{s}}{\sqrt{\left\{\left(R_{s}+R_{r s} / s\right)^{2}+\left(X_{s}+X_{r s}\right)^{2}\right\}}} \text { Amps... } \\
& =\left(\mathrm{I}_{\mathrm{s}(\text { new })}-\mathrm{I}_{\mathrm{oc}}\right) \text { Amps } \ldots \ldots \ldots \ldots \ldots \ldots \ldots \ldots \ldots \ldots \ldots \ldots \ldots \ldots \ldots \ldots
\end{aligned}
$$


2.3 Gross (or Developed) Output Power (Mechanical), $P_{o(G)}$ :

$$
P_{o(G)}=m_{I_{r s}}^{2}\left[R_{r s}(1-s) / s\right] \text { Watt. }
$$

2.4 Maximum Output Power, $P_{\text {o(MAX) }}$ :

$$
\begin{aligned}
& P_{o(M A X)}=m V_{s} \frac{I_{s c(\max )}-I_{o}}{2\left(1+\cos \varphi_{s c}\right)} x_{10}{ }^{-3} \mathrm{~kW} \\
& \cos \varphi_{\mathrm{sc}}=\mathrm{P}_{\mathrm{sc}} / \sqrt{3} \mathrm{xV}_{\mathrm{sc}} \mathrm{x} \mathrm{I}_{\mathrm{sc}} \text { lagging (short-circuit power factor). }
\end{aligned}
$$

2.5 Gross (or Developed) Torque (Mechanical), $\mathbf{T}_{(\mathrm{G})}$ :

$$
\begin{aligned}
& T_{(G)}=P_{0(G)} / \omega_{r}=\frac{m_{I_{r s}}{ }^{2} R_{r s}(1-s) / s}{\left(2 \pi n_{r}\right) / 60} \quad N-m \\
& =\frac{m I_{r s}^{2} R_{r s} / s}{\left(2 \pi n_{s}\right) / 60} \quad N-m
\end{aligned}
$$

2.6 Ratio of Torque at Any Slip to The Pull-Out Torque, $\mathbf{T} / \mathbf{T}_{(\mathrm{PO})}$ :

$$
\begin{aligned}
& T / T_{(P O)}=2 /\left(\frac{s}{s_{p o}}+\frac{s_{p o}}{s}\right) \quad \ldots \ldots \ldots . . . . . \\
& S_{p o} \approx \frac{\left(1+\tau_{1}\right) R_{r s}}{X_{s}+\left(1+\tau_{1}\right) X_{r s}} \quad ; \quad \tau_{l}=X_{s} / X_{m}
\end{aligned}
$$

2.7 Full-Load Power Factor, $\cos \varphi_{\mathrm{rs}}$ :

$$
\cos \phi_{r s}=\frac{R_{s}+R_{r s} / s}{\sqrt{\left[\left(R_{s}+R_{r s} / s\right)^{2}+\left(X_{s}+X_{r s}\right)^{2}\right]}} \quad \text { lagging }
$$

2.8 Open-Circuit Leakage Reactance, $X_{o c}$ :

$$
X_{o c}=\left\{\left(\frac{\sqrt{3} V_{o c}}{I_{o c}}\right)^{2}-\left(\frac{P_{o c}}{I_{o c}^{2}}\right)^{2}\right\}^{1 / 2}=X_{s}+X_{m} \text { ohms. }
$$

2.9 Short-Circuit Leakage Reactance, $X_{\mathrm{sc}}$ :

$$
X_{s c}=\left\{\left(\frac{\sqrt{3} V_{s c}}{I_{s c}}\right)^{2}-\left(\frac{P_{s c}}{I_{s c}^{2}}\right)^{2}\right\}^{1 / 2}=X_{s}+X_{r s} \text { ohms. }
$$

2.10 Short-Circuit Leakage Resistance, $R_{\mathrm{sc}}$ :

$$
\mathrm{R}_{\mathrm{sc}}=\mathrm{P}_{\mathrm{sc}} / \mathrm{I}_{\mathrm{sc}}{ }^{2}=\mathrm{R}_{\mathrm{s}}+\mathrm{R}_{\mathrm{rs}}
$$

\subsection{Power Balance Equations:}

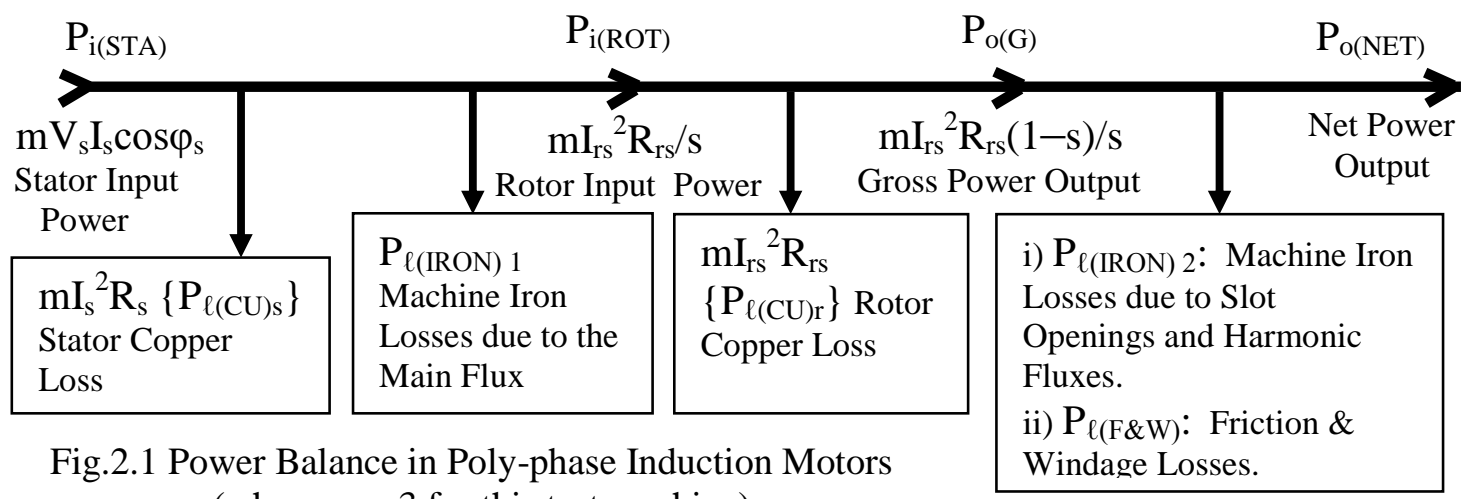
(where $\mathrm{m}=3$ for this test machine). 
The power distribution in a poly-phase induction motor is as shown in Fig.2.1 and from this power balance diagram we have

2.11.1 Net Output Power, $P_{o(N E T)}$ :

$$
\mathrm{P}_{\mathrm{o}(\mathrm{NET})}=\mathrm{P}_{\mathrm{o}(\mathrm{G})}-\left(\mathrm{P}_{\ell(\mathrm{IRON}) 2}+\mathrm{P}_{\ell(\mathrm{F} \& \mathrm{~W})}\right)
$$

2.11.2 Total Fixed Losses, $P_{\ell(F X D) T}$ :

$$
\mathrm{P}_{\ell(\mathrm{FXD}) \mathrm{T}}=\mathrm{P}_{\ell(\mathrm{IRON}) 1}+\mathrm{P}_{\ell(\mathrm{IRON}) 2}+\mathrm{P}_{\ell(\mathrm{F} \& \mathrm{~W})}=\mathrm{P}_{\mathrm{oc}}-\mathrm{mI}_{\mathrm{oc}}{ }^{2} \mathrm{R}_{\mathrm{s}}
$$

2.11.3 Total Motor Losses, $P_{\ell(T O T)}$ :

$$
\mathrm{P}_{\ell(\mathrm{TOT})}=\mathrm{P}_{\ell(\mathrm{FXD}) \mathrm{T}}+\mathrm{P}_{\ell(\mathrm{CU}) \mathrm{T}}=\mathrm{P}_{\ell(\mathrm{FXD}) \mathrm{T}}+\mathrm{P}_{\mathrm{sc}}
$$

2.11.4 Input Power Factor, $\cos \varphi_{s}$,

$$
\cos \varphi_{\mathrm{s}}=\left\{\mathrm{P}_{\mathrm{o}(\mathrm{NET})}+\mathrm{P}_{\ell(\mathrm{TOT})}\right\} / \mathrm{mV}_{\mathrm{s}} \mathrm{I}_{\mathrm{s}}
$$

2.12 Full-Load Efficiency, $\eta$ :

$$
\eta=\mathrm{P}_{\mathrm{o}(\mathrm{NET})} /\left\{\mathrm{P}_{\mathrm{o}(\mathrm{NET})}+\mathrm{P}_{\ell(\mathrm{TOT})}\right\}
$$

2.13 Full-Load Rotor Speed, $n_{r}$ :

$$
\mathrm{n}_{\mathrm{r}}=\mathrm{n}_{\mathrm{s}}\left(1-\mathrm{s}_{\mathrm{fl}}\right)
$$

2.14 Mechanical Loss Torque, $T_{(\text {loss })}$ :

$$
\mathrm{T}_{\text {(loss) }}=60\left(\mathrm{P}_{\ell(\text { IRON }) 2}+\mathrm{P}_{\ell(\mathrm{F} \& \mathrm{~W})}\right) / 2 \pi \mathrm{n}_{\mathrm{s}}
$$

2.15 Delivered Full-Load (Mechanical) Torque, $\mathbf{T}_{(\mathrm{FL})}$

$$
\mathrm{T}_{(\mathrm{FL})}=\mathrm{T}_{(\mathrm{G})}-\mathrm{T}_{\text {(loss) }}
$$

2.16 Ratio of Starting Torque to Delivered Full-Load Torque, $\mathbf{T}_{(\mathrm{ST})} / \mathbf{T}_{(\mathrm{FL})}$ :

$$
\mathrm{T}_{(\mathrm{ST})} / \mathrm{T}_{(\mathrm{FL})}=\left(\mathrm{I}_{\mathrm{s}(\max )} / \mathrm{I}_{\mathrm{rs}}\right)^{2} \mathrm{x} \mathrm{s} \mathrm{s}_{\mathrm{fl}}
$$

\{N.B.: The equations were as obtained from [Shepherd et. al., 1970]; [Kostenko \& Piotrovsky, 1977]; [Daniels, 1976]; [Liwschitz-Garik \& Whipple, 1970]; [Mittle \& Mittal, 1998); [Say, 1976]; $\mathrm{V}_{\mathrm{s}}$ - stator input voltage (nominal); $\mathrm{V}_{\mathrm{oc}}$ - applied open-circuit voltage ; $\mathrm{V}_{\mathrm{sc}}$ - applied short-circuit voltage; $\mathrm{R}_{\mathrm{s}}$ - stator winding resistance; $\mathrm{R}_{\mathrm{rs}}$ - rotor cage resistance referred to stator; $\mathrm{X}_{\mathrm{s}}$ - stator leakage reactance; $\mathrm{X}_{\mathrm{rs}}$ - rotor leakage reactance referred to stator; $\mathrm{X}_{\mathrm{m}}$ - magnetizing reactance; $\mathrm{I}_{\mathrm{s}}$ - stator input current (nominal); $\mathrm{I}_{\mathrm{oc}}$ or $\mathrm{I}_{\mathrm{o}}$ - open-circuit current; $\mathrm{I}_{\mathrm{sc}}-$ short-circuit current on reduced voltage; $\mathrm{I}_{\mathrm{sc}(\max )}$ - short-circuit current on full voltage; $\mathrm{P}_{\mathrm{oc}}-$ open-circuit power dissipation; $\mathrm{P}_{\mathrm{sc}}-$ short-circuit power dissipation; $\cos \varphi_{\mathrm{sc}}-$ short-circuit power factor; $\mathrm{s}$ - any slip; $\mathrm{s}_{\mathrm{fl}}-$ full-load slip; $\mathrm{s}_{\mathrm{po}}-$ slip for the pull-out torque; $\mathrm{m}-$ no. of phases; $\omega_{\mathrm{r}}$ - rotor angular velocity; $\mathrm{T}$ - torque at any slip; $\mathrm{n}_{\mathrm{r}}-$ rotor speed; $\mathrm{n}_{\mathrm{s}}$ - synchronous speed; other terms being as described in Fig.2.1 or in the sub-headings\}.

\section{Laboratory Experimentation Results}

\begin{tabular}{|c|c|c|c|c|}
\hline $\begin{array}{l}\text { TYPE OF } \\
\text { TEST }\end{array}$ & $\begin{array}{c}\text { APPLIED } \\
\text { VOLTAGE }\end{array}$ & $\begin{array}{l}\text { CURRENT } \\
\text { DRAWN }\end{array}$ & $\begin{array}{c}\text { POWER } \\
\text { CONSUMED }\end{array}$ & REMARKS \\
\hline $\begin{array}{l}\text { OPEN- } \\
\text { CIRCUIT }\end{array}$ & $\mathrm{V}_{\mathrm{oc}}=330 \mathrm{~V}$ & $\mathrm{I}_{\mathrm{oc}}=1.2 \mathrm{~A}$ & $\mathrm{P}_{\mathrm{oc}}=360 \mathrm{~W}$ & \multirow[t]{2}{*}{$\begin{array}{l}\text { Motor connected in DELTA. } \\
\text { Tests @ } 35^{\circ} \mathrm{C}\end{array}$} \\
\hline $\begin{array}{l}\text { SHORT- } \\
\text { CIRCIUT }\end{array}$ & $\mathrm{V}_{\mathrm{sc}}=150 \mathrm{~V}$ & $\mathrm{I}_{\mathrm{sc}}=5.4 \mathrm{~A}$ & $\mathrm{P}_{\mathrm{sc}}=720 \mathrm{~W}$ & \\
\hline \multicolumn{3}{|c|}{$\begin{array}{l}\text { STATOR D.C. RESISTANCE TEST, } \\
\mathrm{R}_{\mathrm{s}(\mathrm{DC})}(\text { per-phase })=16.7 \Omega\left[@ 28^{0} \mathrm{C}, \text { Motor Body Temperature. }\right]\end{array}$} & \multicolumn{2}{|c|}{$\begin{array}{l}\text { OPEN-CIRCUIT (OR NO-LOAD) ROTOR SPEED, } \mathrm{n}_{\mathrm{r}}= \\
\text { 1500rpm, as measured with DAWE Stroboscope, Type 1214B }\end{array}$} \\
\hline
\end{tabular}

The Open-Circuit, Short-Circuit and D.C. Resistance Tests were carried out following the usual laboratory procedures. Care was exercised to connect the motor in star (Y) for the D.C. Resistance Test and in delta $(\Delta)$ for the other tests. The results obtained were as presented in Table 3.1 below. However, prior to this, it was necessary to calculate the new input current demand (i.e. stator current for $380 \mathrm{~V}$ ) since the short-circuit current on reduced voltage shall be of the same value. Thus, from equation 2.1 we obtain the following with motor on $\Delta$ connection as required

$$
\mathrm{I}_{\mathrm{s}(\mathrm{new})}=4.9 \mathrm{x}[415 / 380]=5.35 \text { or } 5.4 \mathrm{Amps}=\mathrm{I}_{\mathrm{sc}}
$$

Table 3.1 Open-Circuit, Short-Circuit Test and D.C. Resistance Tests Results

\section{RESULTS AND PERFORMANCE CURVES}

\section{Calculations From Experimentation}

4.1 Calculations from D.C. Test Results:

Stator Resistance, $R_{s(\text { d.c. })}$ and $R_{s(\text { a.c. })}$ [Enyong, 2008]:

$$
R_{s(\text { d.c. })} @ 35 \mathrm{deg} . C=16.7 x \frac{234.5+35}{234.5+28}=17.1 \Omega
$$

But, a.c. resistance should be used. According to [Sadat, 1999], the a.c. resistance at $60 \mathrm{~Hz}$ is about $2 \%$ higher than d.c. resistance. Thus, by simple proportion, it will be smaller at $50 \mathrm{H}_{\mathrm{z}}$ by the factor $(50 / 60)$, ie $0.833 \times 2$ or $1.7 \%$. Thus, 


\subsection{Calculation from the Open-Circuit Test Results:}

$$
R_{s(a . c .)} @ 35 \text { deg.C }=1.017 x 17.1=17.4 \Omega
$$

Combined Stator Leakage and Magnetizing Reactances $\left(X_{s}+X_{m}\right)$, from (2.8):

$$
X_{o c}=\left\{\left(\frac{\sqrt{3} \times 330}{1.2}\right)^{2}-\left(\frac{360}{1.2^{2}}\right)^{2}\right\}^{1 / 2}=X_{s}+X_{m}=405.4 \Omega
$$

4.3 Calculations from the Short-Circuit Test Results:

a) Combined Stator and Rotor Leakage Reactances $\left(X_{s}+X_{r s}\right)$, from (2.9):

$$
X_{s c}=\left\{\left(\frac{\sqrt{3} \times 150}{5.4}\right)^{2}-\left(\frac{760}{5.4^{2}}\right)^{2}\right\}^{1 / 2}=X_{s}+X_{r s}=41.3 \Omega
$$

b) Combined Stator and Rotor Resistances $\left(R_{s}+R_{r s}\right)$, from (2.10):

$$
R_{s c}=720 / 5.4^{2}=R_{s}+R_{r s}=24.7 \Omega
$$

4.4 Other calculations from All the Results Above:

(a) Separation of $X_{s}$ and $X_{r s}$

Taking them to be equal according to [Say, 1976 ], will yield

$$
\mathbf{X}_{\mathrm{s}}=\mathbf{X}_{\mathrm{rs}}=41.3 / 2=\mathbf{2 0 . 7} \mathbf{\Omega}
$$

b) Separating $X_{s}$ and $X_{m}: \mathbf{X}_{\mathbf{m}}=405.4-20.7=\mathbf{3 8 4 . 7} \mathbf{\Omega}$

c) Separating $R_{s}$ and $R_{r s}: \mathbf{R}_{\mathrm{rs}}=24.7-17.4=\mathbf{7 . 3 \Omega}$

d) Effective Load Current, from $(2.2 b)$ : $\mathbf{I}_{\mathrm{rs}}=5.4-1.2=4.2 \mathrm{~A}$

e) Total Fixed Losses, from (2.12): $\mathbf{P}_{\mathbf{\ell}(\mathrm{FXD}) \mathrm{T}}=360-3(1.2 / \sqrt{3})^{2} \times 17.4=\mathbf{3 3 5 W}$

f) Total Machine Losses, $\boldsymbol{P}_{\ell(T O T)}$, from (2.13) and from Fig.2.1:

$$
P_{\mathbf{t}(\text { TOT })}=335+720=1055 \mathrm{~W}
$$

4.5 Calculations based on Parameters derived from Performance Curves 4.5.1 Full-Load Slip $\left(s_{f l}\right)$ from The Current/Slip Curve:

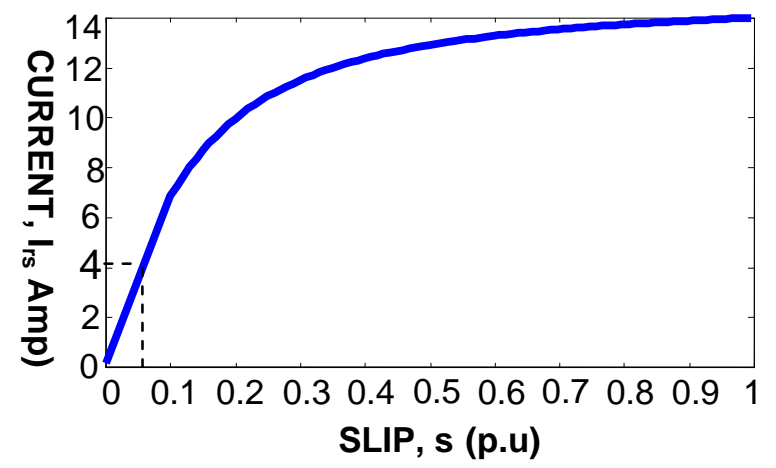

Fig.4.1: Current/Slip Performance Characteristic Curve

The current/slip curve as generated by means of MATLAB using equation 2.2a is as shown in Fig.4.1. The full-load slip, $\mathrm{s}_{\mathrm{fl}}$, being the slip for the current, $\mathrm{I}_{\mathrm{rs}}=4.2 \mathrm{~A}$ as indicated on the curve is 0.053p.u. Based on this value of full-load slip we calculate the following parameters:

a) Full-Load Rotor Speed, $n_{r}$, using (2.16):

Since the rotor speed on no load is virtually the synchronous speed, $\mathrm{n}_{\mathrm{s}}$, because the slip on open circuit is virtually zero according to [Theraja, 1997] and as can be observed from Fig.4.1, we have that $\mathrm{n}_{\mathrm{s}}=1500$ r.p.m. (see Table3.1). Therefore,

$$
\mathbf{n}_{\mathbf{r}}=1500(1-0.053)=\mathbf{1 , 4 2 0 r} . \mathbf{p . m} \text {. }
$$

b) Gross or Developed (Mechanical) Output Power, $P_{o(G)}$, using equation (2.3):

$$
\mathbf{P}_{\mathbf{o}(\mathbf{G})}=3(4.2 / \sqrt{ } 3)^{2} \times 7.3(1-0.053) / 0.053=\mathbf{2 , 3 0 1} \mathbf{W}
$$

c) Friction \& Windage Losses, $P_{\ell(F \& W)}$ :

A typical value of $\mathrm{P}_{\ell(\mathrm{F} \& \mathrm{~W})}$ has been given as $44 \mathrm{~W}$ for a $3 \mathrm{Hp}$ (or $2.24 \mathrm{~kW}$ ) 3-phase cage induction motor at $60 \mathrm{~Hz}$ (see p.146 of [Liwschitz-Garik \& Whipple, 1970]). This amounts to approximately $2 \%$ of the rated 
output power. The same shall be adopted at $50 \mathrm{~Hz}$ for the test machine of $2.2 \mathrm{~kW}$ without attendant significant error for a $10 \mathrm{~Hz}$ difference in frequency. Thus, $\mathbf{P}_{\mathbf{\ell ( \mathrm { F } \& W )}}=\mathbf{4 4 \mathrm { W }}$.

d) Total Iron Losses, $P_{\ell(I R O N) T}$, from (2.12):

$$
\mathbf{P}_{\mathbf{l}_{(\mathrm{IRON}) \mathrm{T}}}=\mathrm{P}_{\ell(\mathrm{FXD}) \mathrm{T}}-\mathrm{P}_{\ell(\mathrm{F} \& \mathrm{~W})}=335-44=\mathbf{2 9 1} \mathrm{W}
$$

e) Iron Losses due to Slot Openings \& Harmonic Fluxes, $P_{\ell(I R O N) 2}$ :

According to [Liwschitz-Garik \& Whipple, 1970]), we can assume that

$$
\mathbf{P}_{\ell(\text { RRON }) 2}=P_{\ell(\operatorname{RON}) 1}=1 / 2 \mathrm{P}_{\ell(\operatorname{RON}) \mathrm{T}}=291 / 2=\mathbf{1 4 5 . 5} \mathbf{W}
$$

f) Gross or Developed (Mechanical) Torque, $T_{(G)}$, from (2.5b):

$$
\mathbf{T}_{(\mathbf{G})}=\frac{3 x(4.2 / \sqrt{3})^{2} \times 7.3 / 0.053}{(2 \times \pi \times 1500) / 60}=\mathbf{1 5 . 4 7 N - m}
$$

g) Mechanical Loss Torque, $T_{\text {(loss) }}$, from (2.16):

$$
\mathbf{T}_{(\text {loss }}=60 \mathrm{x}(145.5+44) / 2 \times \pi \times 1500=\mathbf{1 . 2 1} \mathrm{N}-\mathbf{m}
$$

h) Net or Delivered (Mechanical) Output Power, from (2.11):

$$
\mathbf{P}_{\mathbf{0}(\mathrm{NET})}=2,301-(145.5+44)=\mathbf{2 , 1 1 2} \mathbf{W}
$$

i) Delivered Full-Load (Mechanical) Torque, $T_{(F L)}$, from (2.18):

$\mathbf{T}_{(\mathrm{FL})}=15.47-1.21=\mathbf{1 4 . 2 6} \mathbf{~ N}-\mathbf{m}$

j) Ratio of Full-Load (or Delivered) Torque to The Pull-Out Torque,

$T_{(F L)} / T_{(P O)}$, from $(2.6 a, b)$ :

$$
\begin{aligned}
& \tau_{1}=20.7 / 384.7=0.054 ; \mathrm{s}_{\mathrm{fl}}=0.053 \\
& \mathrm{~S}_{(\mathrm{PO})} \approx(1+0.054) \times 7.3 /\{20.7+(1+0.054) \times 20.7\}=0.18 ; \text { Thus, } \\
& \mathbf{T}_{(\mathbf{F L})} / \mathbf{T}_{(\mathbf{P O})}=2 /\{(0.053 / 0.18)+(0.18 / 0.053)=\mathbf{0 . 5 4}
\end{aligned}
$$

k) The Pull-Out (or Maximum) Torque, $T_{(P O)}$ :

$$
\mathbf{T}_{(\mathrm{PO})}=\mathrm{T}_{(\mathrm{FL})} / 0.54=\mathbf{1 . 8 5 T}_{(\mathrm{FL})}=1.85 \times 14.26=\mathbf{2 6 . 4} \mathbf{~ N}-\mathbf{m}
$$

l) Full-Load Efficiency, from( 2.14): $\boldsymbol{\eta}=2,112 /(2,112+1055)=\mathbf{6 6 . 7 \%}$

m) Full-Load (Output) Power Factor, from (2.7):

$$
\cos \varphi_{\mathrm{rs}}=\frac{17.4+7.3 / 0.053}{\sqrt{\left[(17.4+7.3 / 0.053)^{2}+(41.3)^{2}\right]}}=\mathbf{0 . 9 6 6 ~ L a g g i n g ~}
$$

n) Full-Load Input Power Factor:

$$
\cos \varphi_{\mathrm{s}},=\{2112+1055\} / 3 \times 380(5.4 / \sqrt{ } 3)=\mathbf{0 . 8 9} \text { Lagging }
$$

p) Short-Circuit Power Factor, from (2.4b): $\cos \varphi_{\mathrm{sc}}=720 / \sqrt{3} \times 150 \times 5.4=\mathbf{0 . 5}$ Lagging

4.5.2 Short-Circuit Current on Full Voltage $\left(I_{\text {sc(max }}\right)$ from the Current/Slip Curve:

The value of this short-circuit current is clearly $\mathbf{1 4}$ Amps on the curve being at a slip of unity. Based on this, we are able to further determine the following quantities:

q) Maximum Output Power, $P_{\text {o(MAX) }}$, from (2.4a), using phase current values as due:

$$
\mathbf{P}_{\text {o(MAX) }}=3 x 380 x \frac{(14-1.2) / \sqrt{3}}{2(1+0.5)} x_{10} 0^{-3}=\mathbf{2 . 8} \mathbf{~ k W}
$$

r) Ratio of Starting Torque to Delivered Full-Load Torque, $T_{(S T T)} / T_{(F L)}$, from (2.19):

$$
\mathbf{T}_{(\mathrm{ST})} / \mathbf{T}_{(\mathrm{FL})}=(14 / 4.2)^{2} \mathrm{x} 0.053=\mathbf{0 . 5 9}
$$

4.6 Other Performance Curves generated through MATLAB:

4.6.1 Torque/Speed Characteristic Curve, from (2.5a):

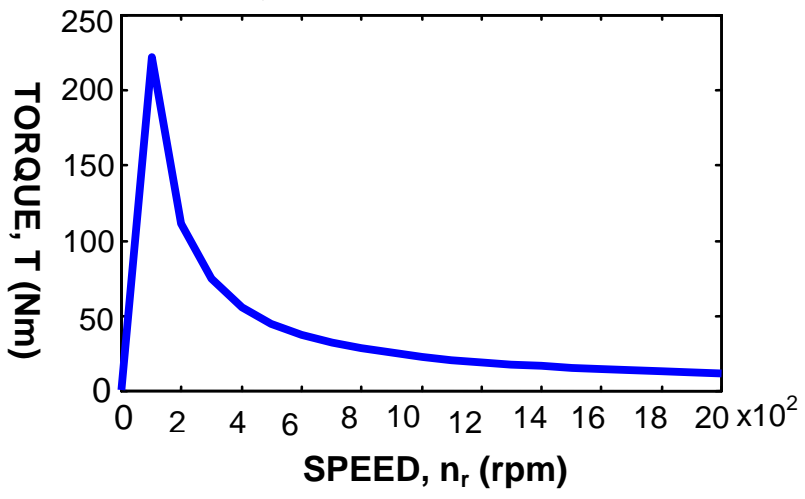

Fig.4.2: Torque/Speed Characteristic of the Test Motor 
4.6.2 Power/Speed Characteristic Curve, from (2.3):

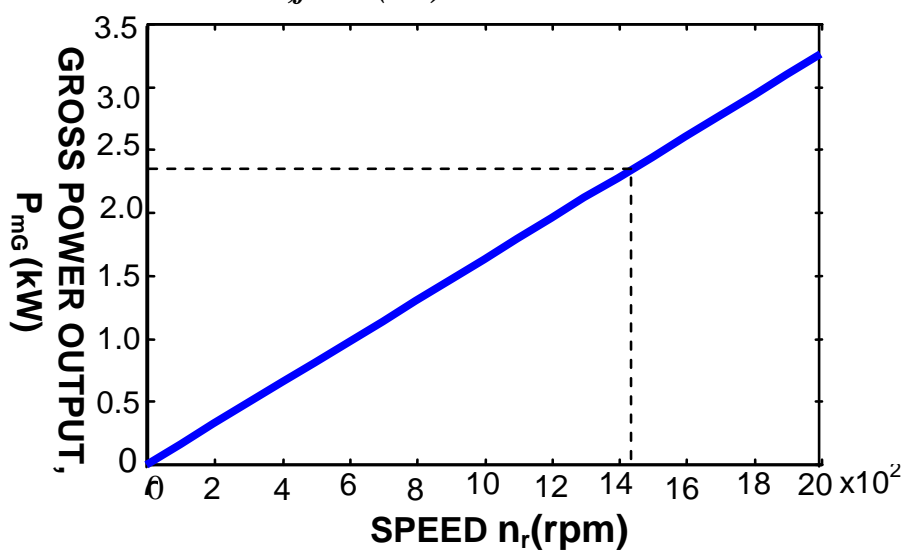

Fig.4.3: Gross Power Output/Speed Characteristic Curve

\section{Analysis, Conclusion \& Recommendation}

\subsection{Performance Analysis}

For the purpose of easy analysis, a summary of the motor parameters obtained from calculations and performance curves shall first be presented as in Table 5.1 below.

Table 5.1: Summary of the Performance Parameters of the Test Motor

\begin{tabular}{|c|c|c|c|c|}
\hline $\mathbf{S} / \mathbf{N}$ & PARAMETER DESCRIPTION & $\begin{array}{c}\text { VALUE } \\
\text { OBTAINED }\end{array}$ & $\begin{array}{c}\text { VALUE } \\
\text { EXPECTED }\end{array}$ & $\begin{array}{c}\text { REMARKS } \\
\text { (On The Expected Values) }\end{array}$ \\
\hline $\begin{array}{l}A \\
\text { i) }\end{array}$ & $\begin{array}{l}\text { LOSSES: } \\
\text { Total Iron Losses }\end{array}$ & $335 \mathrm{~W}$ & $122 \mathrm{~W}$ & $\begin{array}{l}\text { Typical for a } 2.25 \mathrm{~kW} 3 \text {-ph cage motor in } \\
{[1]^{*} \text { p. } 465 \text { and } 3 \mathrm{Hp}(2.24 \mathrm{~kW}) \text { type in [2] }} \\
\text { p.147. }\end{array}$ \\
\hline ii) & \multirow{2}{*}{$\begin{array}{l}\text { Total Copper Losses } \\
\text { Total Machine Losses }\end{array}$} & \multirow{2}{*}{$\begin{array}{l}720 \mathrm{~W} \\
1055 \mathrm{~W}\end{array}$} & $205 \mathrm{~W}$ & $\begin{array}{l}\text { As in [1]; whilst } 210 \mathrm{~W} \text { (a close value) is } \\
\text { the case in [2]. }\end{array}$ \\
\hline 111) & & & $327 \mathrm{~W}$ & Being about $15 \%$ on $2.2 \mathrm{~kW}$ \\
\hline \multirow{2}{*}{$\begin{array}{l}\text { B } \\
\text { i) } \\
\text { ii) }\end{array}$} & \multirow{2}{*}{$\begin{array}{l}\text { ACTIVE POWER: } \\
\text { Net Output Power, } \mathrm{P}_{\mathrm{o}(\mathrm{NET})} \\
\text { Maximum Gross Output } \\
\text { Power, } \mathrm{P}_{\mathrm{o}(\mathrm{MAX})}\end{array}$} & \multirow{2}{*}{$\begin{array}{l}2112 \mathrm{~W} \\
1.3 \mathrm{P}_{\mathrm{o}(\mathrm{NET})}\end{array}$} & $2200 \mathrm{~W}$ & Manufacturer's stated value. \\
\hline & & & $2-2.5 \mathrm{P}_{\mathrm{o}(\mathrm{NET})}$ & $\begin{array}{l}\text { As in [4] p.373; but a typical value in [1] } \\
\text { p.468 is } 1.92 \mathrm{P}_{\text {o(NET). }}\end{array}$ \\
\hline$C$ & $\begin{array}{l}\text { EFFICIENCY: } \\
\text { Full-Load Efficiency, } \eta \text {. }\end{array}$ & $66.7 \%$ & $83 \%$ & $\begin{array}{l}\text { Typical for the motor in [2]; but not lower } \\
\text { than } 81 \% \text { as in [3] p.60. }\end{array}$ \\
\hline \multirow{2}{*}{$\begin{array}{l}D \\
\text { i) } \\
\text { ii) }\end{array}$} & \multirow{2}{*}{$\begin{array}{l}\text { POWER FACTORS: } \\
\text { Full-Load Power Factor } \\
\text { Input Power Factor }\end{array}$} & \multirow{2}{*}{$\begin{array}{l}\text { 0.966Lag } \\
0.89 \mathrm{Lag}\end{array}$} & $0.83 \mathrm{Lag}$ & Standard value as in [3] p.60. \\
\hline & & & $0.748 \mathrm{Lag}$ & $\begin{array}{l}\text { Typical as in [1] p.466; whilst } 0.785 \mathrm{Lag} \text { is } \\
\text { typical in [2] p.147. }\end{array}$ \\
\hline \multirow[t]{2}{*}{$\begin{array}{l}E \\
\text { i) }\end{array}$} & \multirow{2}{*}{$\begin{array}{l}\text { SLIPS: } \\
\text { Full-Load Slip, } \mathrm{s}_{\mathrm{fl}} \text {. } \\
\text { Pull-Out-Torque Slip, } \mathrm{s}_{\mathrm{po}} .\end{array}$} & \multirow{2}{*}{$\begin{array}{l}5.3 \% \\
0.18\end{array}$} & $2-5 \%$ & $\begin{array}{l}\text { Standard as in [4] p. } 370 \text {; but } 2 \% \text { in [1] and } \\
3 \% \text { in [2] are typical. }\end{array}$ \\
\hline & & & $0.12-0.2$ & Standard range as in [5] p.464. \\
\hline \multirow{3}{*}{$\begin{array}{l}\boldsymbol{F} \\
\text { i) } \\
\text { ii) } \\
\text { iii) }\end{array}$} & \multirow{3}{*}{$\begin{array}{l}\text { TORQUES: } \\
\text { Full-Load Torque, } \mathrm{T}_{(\mathrm{FL})} \\
\text { Starting Torque, } \mathrm{T}_{(\mathrm{ST})} \\
\text { Pull-Out Torque, } \mathrm{T}_{(\mathrm{PO})}\end{array}$} & \multirow{3}{*}{$\begin{array}{l}14.26 \mathrm{~N}-\mathrm{m} \\
0.59 \mathrm{~T}_{(\mathrm{FL})} \\
1.85 \mathrm{~T}_{(\mathrm{FL})}\end{array}$} & $11.39 \mathrm{~N}-\mathrm{m}^{* *}$ & Typical value as in [2] p.147. \\
\hline & & & $1.15-1.5 \mathrm{~T}_{(\mathrm{FL})}$ & Standard range as in [2] p.386. \\
\hline & & & $1.6-2.5 \mathrm{~T}_{(\mathrm{FL})}$ & Standard range as in [5] p.482. \\
\hline$G$ & $\begin{array}{l}\text { SPEED: } \\
\text { Full-Load Rotor Speed, } \mathrm{n}_{\mathrm{r}}\end{array}$ & $1,420.5 \mathrm{rpm}$ & $1,420 \mathrm{rpm}$ & Manufacturer's stated value. \\
\hline $\begin{array}{l}H \\
\text { i) } \\
\text { ii) }\end{array}$ & $\begin{array}{l}\text { RESISTANCES: } \\
\text { Stator Resistance, } \mathrm{R}_{\mathrm{s}} \\
\text { Rotor Resistance, } \mathrm{R}_{\mathrm{rs}}\end{array}$ & \multirow{2}{*}{$\begin{array}{l}17.4 \Omega \\
7.3 \Omega \\
\end{array}$} & $\frac{1.46 \Omega}{0.8 \mathrm{R}(\text { at least) }}$ & Typical value as in [1] p.469. \\
\hline \multirow{2}{*}{$\begin{array}{l}\text { I } \\
\text { i) } \\
\text { ii) }\end{array}$} & \multirow{2}{*}{$\begin{array}{l}\text { REACTANCES: } \\
\text { Stator/Rotor Reactances } \\
\text { Magnetizing Reactance }\end{array}$} & & $0.8 \mathrm{R}_{\mathrm{s}}$ (at least) & $0.8425 \mathrm{R}_{\mathrm{s}}=1.23 \Omega$ used in [1]. \\
\hline & & $\begin{array}{l}20.7 \Omega(\text { each }) \\
384.7 \Omega\end{array}$ & $8.1 \Omega$ (each) & Combined value $=16.2 \Omega$ in [1]. \\
\hline
\end{tabular}

\footnotetext{
"Table References: [1] Shepherd et.al.; [2] Liwschitch-Garic \& Whipple; [3] Boehle et. al.;

[4] Mittle \& Mittal; [5] Kostenko \& Piotrovsky; [6] Krause P.C.

${ }^{* *}$ Torque Units Conversion: $8.4 \mathrm{Ib}-\mathrm{ft}=8.4 \times 1.356534=11.39 \mathrm{~N}-\mathrm{m}$.
} 


\subsubsection{Starting Performance:}

It is obvious from item $\mathrm{F}(\mathrm{ii})$ in Table 5.1 that the test machine has a weak starting performance; meaning an inability to start from rest with the full load applied to it, even under direct-on-line starting condition with the stator winding connected in delta.

\subsubsection{Running Performance:}

The machine is seen to have an excellent normal running capability judging from the value of the fullload torque which on the rated speed is $125 \%$ of the expected value. Also, a very good overload capacity which stands at 1.85 is observed; implying that it cannot easily get stalled on reasonable values of overload.

\subsubsection{Efficiency:}

The efficiency on full load is quite poor. This follows the fact that the machine total amount of losses is approximately $245 \%$ of the value $(430 \mathrm{~W})$ appropriate for the acceptable efficiency of $83 \%$ on $2112 \mathrm{~W}$ output. The principal contributory factor is the outrageous value of the stator resistance being roughly 12 times the expected value; meaning that the winding turns per phase is too high and/or the copper wire gauge too small.

\subsection{Conclusion \& Recommendation}

Although the objective of refurbishing the motor to exhibit satisfactory running torques, power factors, slips and speeds was achieved the machine, however, is seen to develop too much heat in the process for a continuous full-load operational duty (i.e. on CMR duty). It is therefore recommended that the motor should be installed and used where it shall be required to render intermittent services only (e.g. as a grinding or drilling machine drive).

\section{References}

[1]. Boehle B. et. al. (1988): Switchgear Manual; Asea Brown Boveri Publication, $8^{\text {th }}$ Ed., Germany.

[2]. Daniels A. R. (1976): Introduction to Electrical Machines, $1^{\text {st }}$ Ed., Macmillan Press Ltd., London.

[3]. Enyong P. M. (2008): Refurbishment and Steady-State Performance Analysis of a 3-phase Induction Motor, M. Eng. Thesis, Elect./Elect. Engng.Dept., University of Benin, Benin-City, Nigeria; p.88-90 .

[4]. Kostenko M and Piotrovsky L. (1977): Electrical Machines, vol. 2, Mir Publishers, Moscow.

[5]. Krause P. C. (1986): Analysis of Electric Machinery; McGraw-Hill Book Company; New York.

[6]. Liwschitz-Garik M. and Whipple C. C. ( --- ): Alternating Current Machines, $2^{\text {nd }}$ Ed., D. Van Nostrand Co. Inc., Princeton, New Jersey.

[7]. Mehala N. (2010): Condition Monitoring \& Fault Diagnosis of Induction Motor Using Motor Current Signature Analysis; PhD Thesis, Electrical Engineering National Institute of Technology, Kurukshetra, India; pp.10-35.

[8]. Mittle V. N. and Mittal A.(1998): Design of Electrical Machines, $4^{\text {th }}$ Ed., Standard Publishers Distributors, Nai Sarak, Delhi.

[9]. Sadat H. (1999): Power System Analysis, Tata McGraw-Hill Publishing Company Ltd., New Delhi.

[10]. Say M. G. (1976): Alternating Current Machines, ELBS $4^{\text {th }}$ Ed., Pitman Publishing Ltd., London.

[11]. Say M. G. and Taylor E. O. (1980): Direct Current Machines, ${ }^{\text {st }}$ Ed., Pitman Publishing Ltd., London.

[12]. Say M. G. (1983): Performance and Design of Alternating Current Machines, CBS Publishers \& Distributors, London.

[13]. Shepherd J. et. al. (1970): Higher Electrical Engineering, $2^{\text {nd }}$ Ed., Pitman Publishing Ltd., London.

[14]. Theraja B. L. (1997): Fundamentals of Electrical Engineering and Electronics, $28^{\text {th }}$ Ed., S Chand \& Co. Ltd., New Delhi. 